\title{
ELEMENTARY EXTENSIONS OF LINEAR TOPOLOGICAL ABELIAN GROUPS
}

\author{
R. G. PHILLIPS AND P. L. SPERRY
}

\begin{abstract}
R. MacDowell and E. Specker obtain a structure theorem for elementary extensions of the integers by considering a certain residue mapping. In this paper we characterize those abelian groups in which an analogous situation exists and obtain the MacDowell-Specker result as a special case of our theory.
\end{abstract}

Introduction. In [3] MacDowell and Specker obtain a structure theorem for elementary extensions ${ }^{*} Z$ of $Z$ ( $Z$ is the additive group of integers). Their method is to construct a homomorphism from ${ }^{*} Z$ to the $Z$-adic completion $\hat{Z}$ of $Z$ which extends the natural embedding $\mu$ of $Z$ into $\hat{Z}$. It turns out that the kernel of this homomorphism is the divisible subgroup $d^{*} Z$ of ${ }^{*} Z$ so that ${ }^{*} Z=d^{*} Z \oplus K$ where $K$ is a subgroup containing $Z$ which is isomorphic to a subgroup of $\mathcal{Z}$ by an isomorphism which extends $\mu$. The homomorphism constructed in [3] essentially sends an element of $* Z$ into its sequence of standard residues modulo $n$ for $n \in N$ ( $N$ is the set of positive natural numbers).

In this paper, we generalize the methods of [3] in the following way. Suppose $G$ is an abelian linear topological group, that is, its topological structure is defined by a filter of subgroups $\mathscr{D}$. Then there are elementary extensions ${ }^{*} G$ of $G$ which extend the topological structure of $G$. We investigate the existence of homomorphisms on these ${ }^{*} G$ into the $\mathscr{D}$ completion $\hat{G}$ of $G$ which extend the canonical map of $G$ into $\hat{G}$ and which are, in some sense, "residue" mappings. According to our Theorem 1 such "residue" mappings exist for every such extension if and only if $\mathscr{D}$ generates a topology coarser than the finite index topology; the mapping defined in I of Theorem 1 is the analog of the mapping defined in [3]. As a corollary of Theorem 1 we obtain a generalization of the Structure Theorem in [3].

Definitions. All groups are abelian; a pair $(G, \mathscr{D})$ will denote a group $G$ and a filter $\mathscr{D}$ of subgroups of $G$. Given $(G, \mathscr{D}), G$ will carry the topology

Received by the editors February 8, 1971.

AMS 1970 subject classifications. Primary 02H20; Secondary 20K45.

Key words and phrases. Elementary extension, linear topological abelian group, completion.

(C) American Mathematical Society 1972 
generated by $\mathscr{D}$ and $\mathscr{D}$ will be written as an indexed family $\left(H_{i}\right)_{i \in I} ; I$ will be partially ordered by: $i \leqq j$ iff $H_{i} \supset H_{j}$. Given $(G, \mathscr{D})$ the completion of $G$ is the projective limit $\hat{G}$ of the system $\left(G / H_{i}, \Pi_{i j}\right)$ where, if $i \leqq j$, $\Pi_{i j}\left(g_{j}+H_{j}\right)=g_{j}+H_{i} ; \hat{G}$ has the relative product topology which has as a base for the neighborhoods of zero the filter $\left(\tilde{H}_{i}\right)_{i \in I}$ where $\left(g_{i}+H_{i}\right)_{i \in I}$ is in $\tilde{H}_{k}$ iff $g_{k}$ is in $H_{k}$. The mapping $\mu$ of $G$ into $\hat{G}$ such that $\mu(g)=\left(g+H_{i}\right)_{i \in I}$ is a continuous homomorphism of $G$ onto a dense subgroup of the complete Hausdorff space $\hat{G}$ (see [1]).

Given $(G, \mathscr{D}), L(G, \mathscr{D})$ will denote a first order language which includes a distinct constant symbol for each element of $G$, a function symbol "+" for the group operation on $G$, and for each $H \in \mathscr{D}$ a predicate symbol $H(x)$ such that $H(a)$ holds in $G$ iff $a \in H$. Let $K(G, \mathscr{D})$ denote all sentences of $L(G, \mathscr{D})$ which hold in $G$; a model of $K(G, \mathscr{D})$ will be called a $\mathscr{D}$-elementary extension of $G$. If ${ }^{*} G$ is a $\mathscr{D}$-elementary extension of $G$ and $H \in \mathscr{D},{ }^{*} H$ will denote the set of all elements $\mathscr{Y}$ of ${ }^{*} G$ which satisfy $H(\mathscr{Y})$ in ${ }^{*} G$. Note that $G$ is a subgroup of $* G$ and ${ }^{*} H \cap G=H$ for all $H \in \mathscr{D}$. A $\mathscr{D}$-elementary extension ${ }^{*} G$ of $G$ will be called a strong $\mathscr{D}$-elementary extension if for each element $\left(g_{i}+H_{i}\right)_{i \in I}$ of $\hat{G}$ there is an element $x$ of ${ }^{*} G$ such that $x-g_{i}$ is an element of ${ }^{*} H_{i}$ for each $i$ in $I$. The topology generated on ${ }^{*} G$ by $\left({ }^{*} H_{i}\right)_{i \in I}$ is called the $S$-topology (see [4]).

Lemma. For any pair $(G, \mathscr{D})$ and any $\mathscr{D}$-elementary extension ${ }^{*} G$ of $G$ there is a strong $\mathscr{D}$-elementary extension ${ }^{* *} G$ of $G$ which is also an elementary extension of ${ }^{*} G$.

Proof. Extend $L(G, \mathscr{D})$ to any first order language $L^{\prime}$ that includes a constant symbol for each element of ${ }^{*} G$. Let $K^{\prime}$ be the set of all sentences of $L^{\prime}$ which hold in ${ }^{*} G$. Extend $L^{\prime}$ to a first order language $L^{\prime \prime}$ by adding, for each $\alpha \in \hat{G}$, a distinct new constant $a_{\alpha}$. For each $\alpha \in \hat{G}$ let $K_{\alpha}=$ $\left\{H_{i}\left(a_{\alpha}-g_{i}\right): i \in I\right\}$ where $\alpha=\left(g_{i}+H_{i}\right)_{i \in I}$. Let $K_{1}=\left(\bigcup\left\{K_{\alpha} \mid \alpha \in \hat{G}\right\}\right) \cup K^{\prime}$. Now for each $\left(g_{i}+H_{i}\right)_{i \in I}$ in $\hat{G}$ and each finite subset $J$ of $I$ there is a $g$ in $G$ such that $g-g_{i}$ is in $H_{i}$ for each $i$ in $J$. From this it is routine to show that $K_{1}$ is consistent. Any model of $K_{1}$ is clearly a strong $\mathscr{D}$-elementary extension of $G$ containing ${ }^{*} G$ as an elementary substructure.

THEOREM 1. For any pair $(G, \mathscr{D})$ the following conditions are equivalent:

I. For every $\mathscr{D}$-elementary extension ${ }^{*} G$ of $G$ there is a homomorphism $\rho$ of ${ }^{*} G$ into $\Pi_{I}\left(G / H_{i}\right)$ which extends $\mu$ and has the property that $\rho(a)=$ $\left(g_{i}+H_{i}\right)_{i \in I}$ implies $a-g_{i} \in * H_{i}$ for all $i \in I$.

II. For every $\mathscr{D}$-elementary extension ${ }^{*} G$ of $G$ there is a homomorphism $\rho$ of ${ }^{*} G$ into $\hat{G}$ which extends $\mu$ and satisfies $\operatorname{Ker} \rho=\bigcap_{I}{ }^{*} H_{i}$ and $\rho\left({ }^{*} H_{i}\right) \subset \tilde{H}_{i}$.

III. $G$ is dense in every strong $\mathscr{D}$-elementary extension ${ }^{*} G$ of $G$ (with respect to the $S$-topology). 
IV. $G$ is dense in every $\mathscr{D}$-elementary extension $* G$ of $G$ (with respect to the $S$-topology).

V. $G / H_{i}$ is finite for all $i \in I$.

Proof. We show $\mathrm{I} \Rightarrow \mathrm{II} \Rightarrow \mathrm{III} \Rightarrow \mathrm{IV} \Rightarrow \mathrm{V} \Rightarrow \mathrm{I}$.

I $\Rightarrow$ II. Let ${ }^{*} G$ be a $\mathscr{D}$-elementary extension of $G$ and let $\rho$ satisfy the property in I. To show that $\rho$ has the property of II we only need prove that $\rho$ maps ${ }^{*} G$ into $\hat{G}$. Given $a \in *^{*} G$ let $\rho(a)=\left(g_{i}+H_{i}\right)_{i \in I}$. Then if $J=$ $\left\{i_{1}, \cdots, i_{n}\right\}$ is any finite subset of $I$ and

$$
X_{J}=\exists x\left[H_{i_{1}}\left(x-g_{i_{1}}\right) \wedge \cdots \wedge H_{i_{n}}\left(x-g_{i_{n}}\right)\right]
$$

then $X_{J}$ holds in $G$ since it holds in ${ }^{*} G$. Thus there is a $g \in G$ so that $g-g_{i_{t}} \in H_{i_{t}}$ for $t=1,2, \cdots, n$ and hence $\rho(a) \in \hat{G}$.

$\mathrm{II} \Rightarrow$ III. Let ${ }^{*} G$ be a strong $\mathscr{D}$-elementary extension of $G$ and let $\rho$ satisfy the property in II. Given $a \in^{*} G$ and $k \in I$ there is an $h \in \tilde{H}_{k}$ and a $g \in G$ such that $\rho(a)+h=\mu(g)$ since $\mu(G)$ is dense in $\hat{G}$. If $h=\left(g_{i}+H_{i}\right)_{i \in I}$, there is a $b \in{ }^{*} G$ such that $b-g_{i} \in^{*} H_{i}$ for all $i \in I$. Let $\rho(b)=\left(t_{i}+H_{i}\right)_{i \in I}$. Since $\rho\left(b-g_{i}\right) \in \tilde{H}_{i}$ for each $i \in I$ we have $g_{i}-t_{i} \in H_{i}$ for all $i$ and hence $\rho(b)=h$. Since $h \in \tilde{H}_{k}, g_{k} \in H_{k}$ and hence $b \in{ }^{*} H_{k} ; a+b-g \in \operatorname{Ker} \rho=\bigcap_{I} * H_{i}$. Therefore $a-g \in{ }^{*} H_{k}$ which proves that $G$ is dense in ${ }^{*} G$.

III $\Rightarrow$ IV. Follows immediately from the lemma.

$\mathrm{IV} \Rightarrow \mathrm{V}$. Suppose $G / H$ is infinite for some $H \in \mathscr{D}$. We set $K^{\prime}=K(G, \mathscr{D}) \cup$ $\{\neg H(\alpha-g): g \in G\}$ where $\alpha$ is a new constant symbol. $K^{\prime}$ is consistent since $G$ serves as a model for any finite subset of $K^{\prime}$ with a suitable interpretation of $\alpha$. But $G$ is not dense in any model of $K^{\prime}$.

$\mathrm{V} \Rightarrow \mathrm{I}$. Let $G / H_{i}=\left\{H_{i}, g_{i_{1}}+H_{i}, \cdots, g_{i_{n}}+H_{i}\right\}$. Then if

$$
X_{i}=\forall x\left[H_{i}(x) \overline{\mathrm{v}} \cdots \overline{\mathrm{v}} H_{i}\left(x-g_{i_{n}}\right)\right]
$$

where " $\bar{v}$ " denotes exclusive "or", $X_{i}$ holds in $G$ for all $i \in I$ and hence holds in every $\mathscr{D}$-elementary extension ${ }^{*} G$ of $G$. Given such a ${ }^{*} G$, if $a \in{ }^{*} G$ we define $\rho(a)=\left(g_{i}+H_{i}\right)_{i \in I}$ if and only if $a-g_{i} \in^{*} H_{i}$ for $i \in I$. Conditions $X_{i}$ assure that $\rho$ is well defined and it follows easily that $\rho$ satisfies the condition in $\mathbf{I}$.

COROLlary. Let $G$ be any group with $H_{n}=n G$ for $n \in N$. If for every elementary extension ${ }^{*} G$ of $G,{ }^{*} G=D \oplus K$ where $D$ is divisible, $G \subset K$ and there is a homomorphism $\phi$ of $K$ into $\hat{G}$ which extends $\mu$, has its kernel contained in $G$ and has a pure image, then I-V are also true.

Proof. The $S$-topology on any ${ }^{*} G$ is the Z-adic topology. Since $\phi[K]$ is pure in $\hat{G}$, the relative and $Z$-adic topologies coincide on $\phi[K]$ so $\mu(G)$ is dense in $\phi[K]$ with respect to the $Z$-adic topology. Hence $\phi[K] / \mu[G]$ is 
divisible and isomorphic to $K / G$ so that ${ }^{*} G / G$ is isomorphic to $D \oplus(K / G)$ which is divisible, hence $G$ is dense in ${ }^{*} G$ and IV holds.

Corollary. Let $G$ be a reduced torsion free group and let $H_{n}=n G$ for $n \in N$. Then $\mathrm{I}-\mathrm{V}$ are equivalent to the property that ${ }^{*} G=d^{*} G \oplus K$ for every elementary extension ${ }^{*} G$ of $G$ where $G \subset K, d^{*} G$ is the divisible subgroup of ${ }^{*} G$ and $K$ is isomorphic to a subgroup of $\hat{G}$ by an isomorphism which extends $\mu$.

Proof. Assume II. The kernel of the $\rho$ guaranteed by II is $\bigcap n^{*} G=$ $d^{*} G$ since ${ }^{*} G$ is torsion free. Since $G$ is reduced and pure in ${ }^{*} G$ there is a subgroup $K$ of ${ }^{*} G$ which contains $G$ such that ${ }^{*} G=d^{*} G \oplus K$. It is easily seen that $\rho \mid K$ satisfies the conditions needed.

Conversely, suppose $\phi: K \rightarrow \hat{G}$ is an isomorphism which extends $\mu$, let $\pi:{ }^{*} G \rightarrow K$ be the projection, then $\phi \pi$ is easily seen to satisfy II since $(n G)^{\sim}=n \hat{G}($ see $[2])$.

Note (see for instance [5, Corollary 2]) that $\Pi J_{p}$, where $J_{p}$ is the $p$-adic integers, is $\hat{Z}$ with respect to the $Z$-adic topology.

Corollary (MacDowell and SPECKER). Any elementary extension of $Z$ is isomorphic to the direct sum of its divisible subgroup and a subgroup of $\Pi J_{p}$.

\section{REFERENCES}

1. N. Bourbaki, Topologie générale, Actualités Sci. Indust., no. 1143, Hermann, Paris, 1960. MR 25 \#4021.

2. L. Fuchs, Infinite abelian groups. I, Pure and Appl. Math., vol. 36, Academic Press, 1970. MR 41 \#333.

3. R. MacDowell and E. Specker, Modelle der Arithmetik, Infinitistic Methods, (Proc. Sympos. Foundations of Math., Warsaw, 1959), Pergamon, Oxford, 1961, pp. 257-263. MR 27 \#2425.

4. A. Robinson, Non-standard analysis, North-Holland, Amsterdam, 1966. MR 34 \#5680.

5. J. Rotman, A completion functor on modules and algebras, J. Algebra 9 (1968), 369-387. MR 37 \#5258.

Department of Mathematics, University of South Carolina, Columbia, South CARolina 29208 